\title{
DISTILLATION CALCULATION BY USE OF CONJUGATED GRADIENT METHOD
}

\author{
TAKaitsu IWATA, Yuichi SATO, Mitsuhiro NAKAMURA \\ AND HIDEO UEMURA \\ Second Plant Engineering Department of Mitsui \\ Shipbuilding and Engineering Co., Ltd., Osaka, 559 \\ IкUно YAMADA* \\ Department of Industrial Chemistry, Nagoya Institute of \\ Technology, Nagoya, 466
}

\begin{abstract}
Distillation calculation by the nonlinear optimization technique is proposed. This method has the following objective function:

$$
\phi(t) \equiv \sum w_{j}\left(X_{j}-1\right)^{2} \longrightarrow \min .
$$

where $t$ denotes the temperature vector, $w_{j}$ the weight factor and $X_{j} \equiv \sum x_{j}(i)$, and $x_{j}(i)$ is obtained by solving the tridiagonal matrix by making the component material balance for a given $t$. The temperature vector is corrected by the conjugated gradient method suggested by Fletcher and Reeves.

From three numerical examples related to the separation of $n \mathrm{C}_{5}$ and $n \mathrm{C}_{6}$ fraction from $\boldsymbol{n} \mathbf{C}_{5} \sim \boldsymbol{n} \mathbf{C}_{9}$ mixture at atmospheric pressure, that of $\mathbf{C}_{2}$ fraction from $\mathbf{C}_{2} \sim \mathbf{C}_{3}$ mixture at high pressure and extractive distillation, it has been confirmed that the proposed method provides a stable solution as compared with the matrix methods by Amundson et al. and Saito et al., it retains the simplicity of the matrix method, and the storage required for the main array in this method is obviously less than that for the original gradient method by Naphtali et al.
\end{abstract}

The distillation process is a well-known unit operation to separate liquid mixtures and is widely used in the chemical and petroleum industries. Useful calculation methods to solve the operation problem for distillation may be essentially classified into the following items: matrix method ${ }^{1,11}, \theta$-method ${ }^{6}$, and relaxation method $^{8)}$. Some attempts have recently been made to improve practical use both by combining matrix method with $\theta$-method and by modifying the method itself with the advantage of electronic computer technics. Some attempts have been reviewed by several investigators ${ }^{5,12)}$. In this paper a new approach using the conjugated gradient method, which provides the stable solution to the mass and heat balance equations, is proposed. This method retains simplicity as well as the Thomas algorithm used in the ordinary matrix method, and may be applicable to similar stagewise operations.

\section{Formulation of Problem}

For simplicity an ordinary distillation column with single-feed plate and without any side-cut streams is treated as the model column, and the feed is supplied as saturated liquid. Let us number the plates down from the top of column, so that the total condenser, the feed plate and the reboiler are assigned

Received March 7, 1975. the symbols $1, f$ and $N$, respectively. The fundamental equations for solving distillation problems are derived by making material and heat balances around $j$-th plate of the model.

Overall material balance:

$$
L_{j-1}-\left(V_{j}+L_{j}\right)+V_{j+1}=-F_{j}
$$

Component material balance:

$$
\begin{gathered}
L_{j-1} x_{j-1}(i)-\left(V_{j} y_{j}(i)+L_{j} x_{j}(i)\right)+V_{j+1} y_{j+1}(i) \\
=-F_{j} x_{F j}(i)
\end{gathered}
$$

Heat balance:

$$
L_{j-1} h_{j-1}-\left(V_{j} H_{j}+L_{j} h_{j}\right)+V_{j+1} H_{j+1}=-F_{j} h_{j}
$$

where $F_{j} x_{j}=0, F_{j} h_{j}=0$ at $j \neq f, F_{j} x(i)=F x_{F}(i)$ and $F_{j} h_{j}=F h_{F}$ at $j=f$. On the other hand, the relationship between vapor and liquid compositions can be expressed by

$$
y_{j}(i)=K_{j}(i) x_{j}(i)
$$

where the assumption of ideal stage is used. Substituting Eq. (4) into Eq. (2), the component material balance is given by the following tridiagonal matrix:

$$
\boldsymbol{M x}=\boldsymbol{F}
$$

where $\boldsymbol{x}^{T} \equiv\left[x_{1}(i) \ldots x_{f}(i) \ldots x_{N}(i)\right]^{T}$ and $\boldsymbol{F} \equiv\left[0 \ldots-F x_{F}\right.$ (i)...0 $]^{T}$ and the elements of $\boldsymbol{M}$ are as follows:

$$
\begin{aligned}
& m_{j j} \equiv-\left(V_{j} K_{j}(i)+L_{j}\right) \\
& m_{11} \equiv-\left(L_{1}+D\right)
\end{aligned}
$$




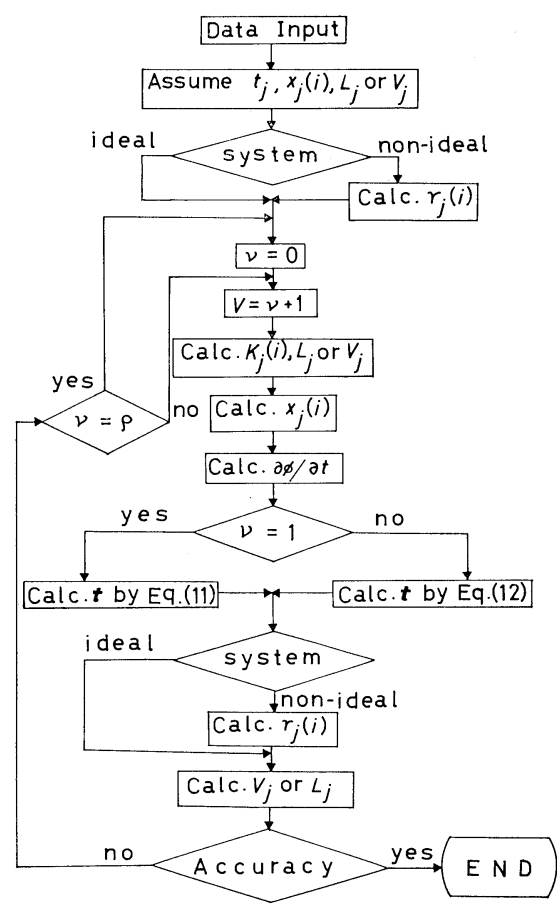

Fig. 1 Flow chart of calculation procedure

$$
\begin{aligned}
& m_{N N} \equiv-\left(V_{N} K_{N}(i)+W\right) \\
& m_{j, j-1} \equiv L_{j-1} \\
& m_{j, j+1} \equiv V_{j+1} K_{j+1}(i)
\end{aligned}
$$

Eq. (5) is a nonlinear simultaneous equation with respect to $x_{j}(i)$ and $t_{j}$, but this equation can be regarded as a linear equation if the element $m_{j, k}(k=$ $j-1, j, j+1)$ is given by a constant, which can be solved by the Thomas method. This is the first characteristic of the original matrix method ${ }^{1}$. However, the elements are affected by the temperature profile in the column. Therefore, the relationships $\sum x_{j}(i)=1$ and $0<x_{j}<1$ for all $j$ are unsatisfied unless the profile is given exactly. Then, $x_{j}(i)$ is corrected by the treatment of normalization, and the elements $m_{j, k}$ are modified by calculating the bubble point with normalized $x_{j}(i)$ and by calculating Eq. (3) with new values of $H_{j}^{V}$ and $h_{j}^{L}$ obtained from the normalized $x_{j}(i)$. This procedure is the second characteristic of the original matrix method. But it can be shown that when the liquid mixture has nonideality this method will fail to converge in general. Saito et al. ${ }^{9)}$ improved this method by using the concept of perturbation theory to correct the component activity coefficient. Tomich $^{10)}$ and Naphtali et al. ${ }^{7)}$ proposed the matrix method with Newton-Raphson method to solve the material and the heat balances simultaneously.

To obtain a stable solution for the distillation problem, the conjugated gradient method by Fletcher and Reeves $^{3)}$ is herein applied.

Let us now define an objective function $\phi(t)$ as follows:

$$
\phi(\boldsymbol{t})=\sum w_{j}\left(X_{j}-1\right)^{2}
$$

where $X_{j} \equiv \sum_{j} x_{j}(i), \boldsymbol{t}$ is the liquid temperature vector and $w_{j}$ denotes a weight factor for plate $j$. It is considered that the value of $\phi(t)$ must be zero when the calculation has converged with an infinite number of significant figures. Since the actual calculation is carried out by several number of significant figures, $\phi(t)$ has a finite value which depends upon the accuracy of calculation. Generally, $\phi(t)$ has a single peak around the solution.

Based on the consideration mentioned above, it will be concluded that to solve the distillation problem can be reduced to solving the nonlinear optimization problem with the following objective function:

$$
\left\{\begin{array}{l}
\phi(\boldsymbol{t})-\text { min. } \\
\text { Subject to Eqs. (3) and (5) }
\end{array}\right.
$$

\section{Calculation Procedure}

The calculation procedure of the present method is summarized by the flow chart shown in Fig. 1. The algorithm of the conjugated gradient method for correcting the temperature vector, $\boldsymbol{t}$, can be shown as follows:

For $\nu$-th calculation step, the temperature gradient vector with respect to $\phi(\boldsymbol{t})$ is written as

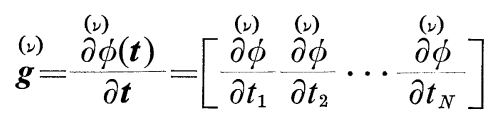

Hence, the temperature vector is corrected by

$$
\stackrel{(\nu)}{t}={ }^{(\nu-1)} \boldsymbol{t}+\lambda_{\mathrm{opt}}^{(\nu-1)}
$$

where $\boldsymbol{d}$ is calculated by the following equations.

For initial step:

$$
\stackrel{(1)}{\boldsymbol{d}}=-\stackrel{(1)}{\boldsymbol{g}}
$$

where this equation implies that $t$ is corrected in the direction of the maximum gradient

For general routine:

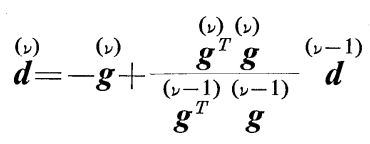

where this equation shows that $t$ is corrected in the direction of the conjugated gradient.

According to the original conjugated gradient method $^{3)}$, the calculation of Eq. (12) is repeated until $\nu=N^{\prime}$, where $N^{\prime}$ shows number of variables for general problem. In this work, however, as suggested in Fig. 1, maximum value of $\nu$ is given by a finite number $\rho$ which is less than $N^{\prime}$, to eliminate the truncation error in Eq. (12), then, for $\nu=\rho+1$ the step returns at $\nu=1$. Furthermore, $\lambda_{\text {opt }}$ in Eq. (10) to accelerate and stabilize the convergence is obtained by minimum values of the equation 


$$
\Omega(\lambda)=\phi\left({ }^{(\nu-1)}{ }^{(\nu-1)}+\lambda d\right) \longrightarrow \min .
$$

where the golden section method is useful to obtain $\lambda_{\text {opt }}$. The graphical meaning of the presentation of $\lambda_{\text {opt }}$ is shown in Fig. 2 for two variables of $t_{j}$.

This procedure is repeated until the convergence function used in the distillation calculation has converged within a certain accuracy.

The procedure to obtain $\partial \phi / \partial t_{k}$ is as follows: The differentiation of Eq. (7) gives

$$
\frac{\partial \phi}{\partial t_{k}}=\frac{\partial}{\partial t_{k}}\left[\sum_{j} w_{j}\left(X_{j}-1\right)^{2}\right]=2 \sum_{j}\left\{w_{j}\left(X_{j}-1\right) \sum_{i} \frac{\partial x_{j}(i)}{\partial t_{k}}\right\}
$$

Furthermore, the calculation of $\partial x_{j}(i) / \partial t_{k}$ proceeds below. The differentiation of Eq. (5) leads to the following tridiagonal matrix.

$$
\boldsymbol{M} \dot{\boldsymbol{x}}=\boldsymbol{z}
$$

where the element in $\boldsymbol{M}$ is already in Eq. (6). $\boldsymbol{x}, \boldsymbol{z}$ and the elements in $z$ are given as follows:

$$
\begin{aligned}
& \dot{x} \equiv\left[\partial t_{k}(i) / \partial t_{k} \partial x_{2}(i) / \partial t_{k} \cdots \partial x_{N}(i) / \partial t_{k}\right]^{T} \\
& z \equiv\left[z_{1 k} z_{2 k} \cdots z_{N k}\right]^{T} \\
& z_{11}=0 \\
& z_{k, k}=V_{k} x_{k}(i) \frac{d k_{k}(i)}{d t_{k}} \\
& z_{k-1, k}=-V_{k} x_{k}(i) \frac{d k_{k}(i)}{d t_{k}} \\
& z_{j, k}=0 \text { for } j<k-2, j>k+1
\end{aligned}
$$

where $d k_{k}(i) / d t_{k}$ can be calculated by the differentiation of Cox-Antoine's equation if the concept of perturbation theory ${ }^{9}$ is applied to the component activity coefficient. For a given set value of $t_{j}, V_{j}$ and $L_{j}$ are calculated from Eq. (3) with the value of $H^{V}$ and $h^{L}$ obtained by the previous step, and $\partial x_{j}(i) /$ $\partial t_{k}$ can be obtained by solving Eq. (15). Then, $\stackrel{(\nu)}{g}$ is calculated by Eq. (9). Thus, the temperature vector is corrected by Eq. (10).

\section{Discussion and Conclusion}

To examine the proposed method, three numerical examples for calculating $x_{D}(i)$ and $x_{W}(i)$ under the given operating condition are illustrated.

The first example given in Table 1 is related to the separation of $n \mathrm{C}_{5}$ and $n \mathrm{C}_{6}$ fractions from $n \mathrm{C}_{5} \sim n \mathrm{C}_{9}$ mixture at atmospheric pressure. Under the given condition of convergence, $\left|X_{j}-1\right|_{\max }<10^{-3}$, the solution, number of iteration and C.P.U. time required by use of I.B.M. 370-168 are given in Table 2, where $w_{j}$ is unity for all $j$. For this example much

\begin{tabular}{|c|c|c|c|c|c|c|}
\hline \multicolumn{2}{|c|}{ Component } & $i$ & $x_{F}(i)$ & $A(i)$ & $B(i)$ & $C(i)$ \\
\hline \multicolumn{2}{|c|}{$n \mathrm{C}_{5}$} & (1) & 0.25 & 2.15752 & 221.383 & 65.755 \\
\hline \multicolumn{2}{|c|}{$n \mathrm{C}_{6}$} & (2) & 0.25 & 2.06306 & 234.633 & 51.669 \\
\hline \multicolumn{2}{|c|}{$n \mathrm{C}_{7}$} & (3) & 0.20 & 2.18817 & 338.723 & 67.551 \\
\hline \multicolumn{2}{|c|}{$n \mathrm{C}_{8}$} & (4) & 0.15 & 2.34773 & 462.208 & 82.981 \\
\hline \multicolumn{2}{|c|}{$n \mathrm{C}_{9}$} & (5) & 0.15 & 2.53973 & 610.673 & 99.707 \\
\hline$F=1.0$ & $D=0.5$ & $R=2.0$ & $N=$ & $=12 f=6$ & $q=1.0$ & $\Pi=1.0$ \\
\hline \multicolumn{7}{|c|}{ Table 2} \\
\hline$i$ & $x_{D}(i)$ & $x_{W}(i)$ & & & $\begin{array}{l}\text { This } \\
\text { method }\end{array}$ & $\begin{array}{l}\text { Mat. } \\
\text { method }\end{array}$ \\
\hline (1) & 0.5000 & - & & & & \\
\hline (2) & 0.4909 & 0.0093 & No & of iteration & ns 17 & 35 \\
\hline (3) & 0.0091 & 0.3907 & & & & \\
\hline (4) & - & 0.3000 & C.P. & U. Time 2 & $2.81 \mathrm{sec}$ & $1.15 \mathrm{sec}$ \\
\hline (5) & - & 0.3000 & req & ired & & \\
\hline
\end{tabular}
more C.P.U. time for the proposed method is required as compared with the primitive matrix method ${ }^{1}$.

\begin{tabular}{|c|c|c|c|c|c|c|c|}
\hline \multicolumn{2}{|c|}{ Component } & $i$ & $x_{F}$ & & $A(i)$ & $B(i)$ & $C(i)$ \\
\hline \multicolumn{2}{|c|}{$\mathrm{C}_{2} \mathrm{H}_{4}$} & (1) & 0.6 & 4339 & 2.48956 & 173.497 & 181.642 \\
\hline \multicolumn{2}{|c|}{$\mathrm{C}_{2} \mathrm{H}_{6}$} & (2) & 0.1 & 2805 & 2.48604 & 187.010 & 173.162 \\
\hline \multicolumn{2}{|c|}{$\mathrm{C}_{3} \mathrm{H}_{4}$} & (3) & 0.0 & 0141 & 2.93288 & 455.269 & 215.704 \\
\hline \multicolumn{2}{|c|}{$\mathrm{C}_{3} \mathrm{H}_{6}$} & (4) & 0.2 & 1634 & 2.47325 & 226.453 & 155.943 \\
\hline \multicolumn{2}{|c|}{$\mathrm{C}_{2} \mathrm{H}_{8}$} & (5) & 0.0 & 1031 & 2.45346 & 234.968 & 158.263 \\
\hline \multicolumn{8}{|c|}{$F=1.0 D=0.7725 \quad R=3.0 \quad N=18 f=9 q=1.0 \quad \Pi=27.7 \mathrm{~atm}$} \\
\hline \multicolumn{8}{|c|}{ Table 4 Solution of numerical example 2} \\
\hline$i$ & $x_{D}(i)$ & \multicolumn{3}{|c|}{$x_{W}(i)$} & & $\begin{array}{l}\text { This } \\
\text { method }\end{array}$ & $\begin{array}{c}\text { Mat. } \\
\text { Method }\end{array}$ \\
\hline (1) & 0 . & \multicolumn{3}{|c|}{0.0008} & & & \\
\hline (2) & 0.1651 & & 024 & \multicolumn{2}{|c|}{ No. of iteration } & 17 & 122 \\
\hline (3) & - & & 062 & & & & \\
\hline (4) & 0.0016 & & 454 & \multicolumn{2}{|c|}{ C.P.U. Time } & $4.68 \mathrm{sec}$ & $4.87 \mathrm{sec}$ \\
\hline (5) & - & & 452 & & & & \\
\hline
\end{tabular}

The second example shown in Table 3 is related to the separation of $\mathrm{C}_{2}$ fraction from $\mathrm{C}_{2} \sim \mathrm{C}_{3}$ mixture at

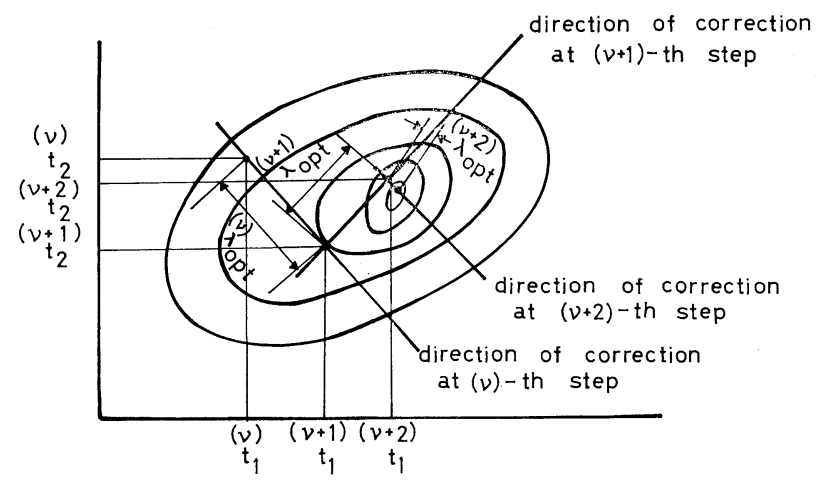

Fig. 2 Graphical presentation of $\lambda_{\mathrm{opt}}$ for two variables of $t_{j}$

Table 1 Statement of numerical example 1

Table 3 Statement of numerical example 2

high pressure, where the Cox-Antoine constants, $A(i), B(i)$ and $C(i)$ are correlated by using the $K$ value estimated from the method proposed by Chao and Seader $^{2)}$. Under the same condition of convergence and $w_{j}$ as in the first example, the solution is given in Table 4. The C.P.U. time required for the proposed method is almost the same as that for the primitive matrix method ${ }^{1)}$.

The third example is related to the extractive distillation shown in Table 5, where constant molar rates of vapor and liquid are assumed. In this ex- 


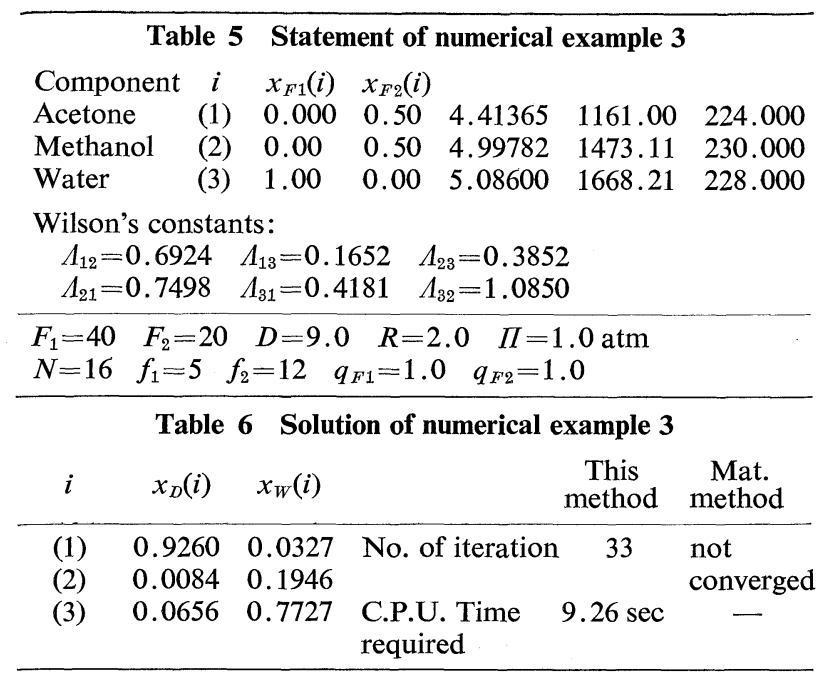

ample, Eqs. (1) (3) are corrected as follows:

$$
\begin{array}{ll}
F_{j} \neq 0 & \text { at } j=f_{1}, f_{2} \\
F_{j} x_{j}(i)=F_{f 1} x_{F f 1}(i), \quad F_{f 2} x_{F f 2}(i) & \text { at } f=f_{1}, f_{2} \\
F_{j} h_{j}=F_{f 1} h_{f 1}, \quad F_{f 2} h_{f 2} &
\end{array}
$$

where suffix $f_{1}$ denotes the main feed and $f_{2}$ the 'solvent feed. For the same condition of convergence as in the examples 1 and 2, the solution is given in Table 6, where the iteration method by use of the concept of perturbation theory ${ }^{9)}$ has been applied to correct the component activity coefficient. Also, the variation of molar rate of liquid throughout the column has been considered to evaluate $w_{j}$ in each section. In this example the solution by use of the matrix method $^{9)}$ has not converged.

As observed in the solutions mentioned in Tables 2, 4 and 6, it can be shown that the proposed method has the remarkable advantage of a stable solution with a small number of iteration steps as compared with other matrix methods ${ }^{1,9}$.

It is well-known that the matrix method with Newton-Raphson method is useful to solve nonlinear simultaneous equations. Therefore, several investigators $^{7,10)}$ have proposed the socalled gradient method to solve Eq. (5). This method, however, is not certain to obtain a stable solution in general when the variable increases. So some attempts ${ }^{4}$ have been made to improve the original gradient method, whereas the present method provides a stable solution even when the number of components and theoretical plates increase. Moreover, in this work the storage required for the main array can be shown as follows:

$\begin{array}{lc}h_{j} & N \\ H_{j} & N \\ y_{j}(i) & N \times C_{n} \\ x_{j}(i) & N \times C_{n} \\ \gamma_{j}(i) & N \times C_{n} \\ K_{j}(i) & N \times C_{n} \\ P_{j}(i) & N \times C_{n}\end{array}$

\begin{tabular}{lc}
$t_{j}$ & $N$ \\
$V_{j}$ & $N$ \\
$L_{j}$ & $N$ \\
$\partial \phi / \partial t_{k},(\boldsymbol{\nu})(\boldsymbol{d}, \boldsymbol{g}, \stackrel{(\nu-1)}{\boldsymbol{g}})$ & $3 N$ \\
$X_{j}$ & $N$ \\
\hline Total & $N\left(5 C_{n}+9\right)$
\end{tabular}

where $C_{n}$ denotes the number of components. Thus, the total storage required in this work is obviously less than $N\left(2 C_{n}^{2}+9 C_{n}+6\right)$, which is required in the original gradient method ${ }^{7}$.

\section{Nomenclature}

$A(i), B(i), C(i)=$ constants of component $i$ in CoxAntoine's equation, $\log P(i)=A(i)-B(i) /$ $(t+C(i))$

$=$ molar rate of distillate

$D$
(L)

$=$ direction vector for correction of temperature given by Eqs. (11) and (12) at $\nu$-th convergence step

$=$ vector of molar rate of feed

$=$ molar rate of feed

$=$ temperature gadient vector for $\phi$ given by Eq. (9) at $\nu$-th convergence step

$=$ enthalpy of vapor phase

$\begin{array}{ll}h & =\text { enthalpy of liquid phase } \\ K(i) & =K \text {-value of component } i\end{array}$

$L \quad=$ liquid molar rate

$\boldsymbol{M}=$ tridiagonal matrix of Eq. (5) and (15) with elements given by Eq. (6)

$m_{i j} \quad=$ elements given by Eq. (6)

$N \quad=$ total number of plates including total condenser and reboiler

$P(i) \quad=$ vapor pressure of component $i$

$q \quad=$ thermal factor of feed

$R \quad=$ reflux ratio

$\boldsymbol{t}=$ temperature vector

$t \quad=$ temperature of liquid

$V \quad=$ molar rate of vapor

$W \quad=$ molar rate of bottoms

$w \quad=$ weight factor in Eq. (7)

$X \quad=\equiv \sum x(i)$

$\dot{\boldsymbol{x}} \quad=$ vector of temperature gradient for $x(i)$ with the elements given by Eq. (16)

$\boldsymbol{x}=$ vector of liquid composition

$x(i) \quad=$ molar fraction of liquid of component $i$

$y(i)=$ molar fraction of vapor of component $i$

$\boldsymbol{z} \quad=$ vector in Eq. (15)

$z_{i j} \quad=$ elements given by Eq. (18)

$\gamma(i)=$ activity coefficient of component $i$

$\Lambda \quad=$ Wilson's constant

$\lambda \quad=$ parameter in Eq. (13)

$\nu \quad=$ number of iterations

$\Pi=\quad$ total pressure

$\rho \quad=$ parameter to eliminate the truncation error in Eq. (12)

$\phi \quad=$ objective function given by Eq. (7)

$\Omega \quad=$ function given by Eq. (13)

$\langle$ Subscripts〉

$F \quad=$ feed

$f \quad=$ feed plate

$j, k=j$-th and $k$-th plates 


\section{Literature Cited}

1) Amundson, N. R. and A. J. Pontinen: Ind. Eng. Chem., 50, 730 (1955).

2) Chao, K. C. and J. P. Seader: AIChE J., 7, 98 (1961).

3) Fletcher, R. and C. M. Reeves: Computer Journal, 7, 149 (1964).

4) Hirose, Y. and M. Kudo: Preprint for 8th Autumn Meeting of The Soc. of Chem. Engrs. Japan, B-215, Tokyo (1974).

5) Holland, C. D.: "Multicomponent Distillation" PrenticeHall Inc. (1963).

6) Lyster, W. N. and C. D. Holland: Petorol Refiner, 41, 206
(1959).

7) Naphtali, L. M. and D. P. Sandholm: AIChE J., 17, 148 (1971).

8) Rose, A.: Ind. Eng. Chem., 50, 737 (1958).

9) Saito, H., H. Wada, S. Kanno and A. H. Sugie: J. Chem. Eng. Japan, 7, 267 (1974).

10) Tomich, J. J. F.: AIChE J., 16, 229 (1970).

11) Wang, J. C. and C. E. Henk: Hydrocarbon Processing, 45, 155 (1966).

12) Yamada I.: “Advanced Chemical Engineering”, Vol. 7, p. 95, Maki Shyoten (1973). 